\title{
Medical-patient relationship; doctors and patients, patients and doctors; disease and illness
}

\section{Opinion}

The relation medical doctor/patient are today's big challenge. The internet is a new participant in this relation. The medical doctor should learn empathy, complicity, an appropriate language and close relation. There should be a preparation for the interview bad news. The author expresses herself how to optimize the relation medical doctor/patient.

The doctor patient relationship is a relation based on trust, in an appropriate language as a fundamental part of medical practice, totally related to the clinical method and linked to the classic bioethical principles of: Autonomy, Beneficence, Non-Maleficence, Secrecy and Justice Because we treat people and not diseases. In Portuguese when the word disease is mentioned it is applied in a broad sense. It contains psychological implications terms, which include the subjective response of the patient to this fact. However, we do not discriminate the double meaning the concept involves, which necessarily separates the disease burden by itself. In practice this dichotomy means that illness or disease is what leads the patient to the doctor, being the disease what the patient feels after the medical consultation and verdict.

The patient's illness and the medical illness are terms used to describe what the patient feels when he goes to the doctor and what he is diagnosed with when he gets home after the consultation and verdict. It was Casser ${ }^{1}$ who introduced this double sense in which disease is something related to the organ and illness affection is something the person has. The illness will then be the subjective response of the individual and those around him towards an episode of illness and how they interpret the origin and meaning of this event, that is, how their behavior and their relationship with others will be developed and what should be done to resolve the situation.

When the patient looks up for the doctor, he already has a set of ideas about his health problem from the cause to the consequences, from gravity to desirable treatments, and may even have consulted other doctors, and if these previous ideas are not explored and discussed during the visit, there is a high probability that the patient will not adhere to the proposed treatment and feel dissatisfied and frustrated with the clinical encounter. The way the relation is established is what will modulate the relationship and change the possible legal ethical implications of a bad outcome.

How can we then align the respect for proper information and the modulation of this same information with the profile of the patient, when the time and conditions in which the encounter takes place are often subject to noise phenomena that bias the relationship? The 'disease' and 'illness' do not necessarily coexist. There can be disease without ailment and ailment without disease. This is the case such as of ocular hypertension situation or glaucoma or diabetic retinopathy even without visual repercussions, as the patient does not have any symptoms. One must try to understand how the patient perceives the new episodes of illness identified by the doctor, how it affects his behavior and relationships with other members, what he is willing to change and what he will do to deal with the situation, and not
Volume 3 Issue 4 - 2019

\author{
Leonor Duarte Almeida,' Pedro Gehl Braz,' \\ 'Department of Ophthalmology, Medicine University of Lisbon, \\ Portugal \\ ${ }^{2}$ Lisboa e Vale do Tejo.ACES Estuário do Tejo USF Forte
}

Correspondence: Leonor Duarte Almeida, Department of ophthalmology, Medicine University of Lisbon, Portugal, Tel 00351962918026,Email leonorduartealmeida@gmail.com

Received: May 21, 2019 | Published: June 25, 2019

delegate it to family members. The family is supportive but does not replace the patient who may even prefer their non-involvement. There are explanatory models that teach about the disease, its severity, its cause, its treatment and guide the choices between different available therapies, giving personal and social meaning to the experiences of illness. The meeting between the doctor and the patient is also a meeting of two different explanatory models, the one of the patient and the one of the doctor, and if these models are not explained, discussed and negotiated, this implies that the doctor's message is not understood or it is not accepted by the patient who decides accordingly, not to follow the doctor's advice. ${ }^{2}$ Only a small percentage of people with abnormal symptoms consult the doctor and when they do only $1 / 3$ completely follows the prescriptions, $1 / 3$ follows them partially and $1 / 3$ do not follow them at all. This rule called $1 / 3$ Podell was confirmed by several studies and poses the problem of medical / patient communication and its implications for adherence to therapy. ${ }^{2,3}$ In order to explain this phenomenon Kleinman proposes the said theory of 'explanatory models', as ideas about an episode of disease and its treatment used by those involved in a clinical process. ${ }^{2}$ For example, I myself never write on the computer when observing the patient. I look directly into his/her eyes and listen carefully their complaints before registering all the data. I consider this a good way of establishing a close relation with the patient acquiring empathy and respect. For me these are also a first way of being responsible for the other, and clarify the patient's ideas and expectations.

This initial clarification of the patient's ideas and expectations, both regarding him and the physician's performance, is fundamental for a better negotiation of the plan and agreement with the objectives and goals to be achieved. The reason for the consultation, expressed by the patient and his narrative is the key to the solution, thereby exploring his motive and his previous ideas about his problem and discuss them during the consultation end up to be decisive to understand why the patient looked up for the health services. It is not the symptom that matters but what the patient "thinks" about it. ${ }^{4}$ The reason for the consultation is only the password for accessing health care and the interpretation that the patient and the doctor make determines the whole subsequent process of care delivery. ${ }^{4}$ It should be noted that when the patient seeks the doctor he already has a set of ideas about his health problem. Fundamental is never to forget the motive that has led you to seek health care, be it an explicit one or a hidden one. 
If this is not valued, if the patient's previous ideas about his problem are not explored and discussed, there is a risk of the doctor getting lost in the new problems he identified and, consequently, the patient being dissatisfied with the clinical encounter, may lose an opportunity to create an alliance. ${ }^{5}$ The doctors who just wrote about the disease lost an opportunity to explain how medical training is fundamentally aimed at the pathophysiology, clinic, evaluation of the complementary diagnostic tests, having devaluated the patient's narrative and the moral and eventually psychological support that he may have had needed. For this reason it should be given a special focus at the physician's professional behavior in knowing the patient's reality, his capability of listening to the complaints and his ability to propose strategies that will help him adapt to the limits induced by the disease, which may not exactly mean that the health professional has to have a deep understanding on other areas of knowledge, such as those of a psychological or psychoanalytical forum.

For Kleinman ${ }^{6}$ about half of the patients in the United States who look for a general practitioner say they feel frustrated as they realize their symptoms and identify them as relevant in order to help identify the diagnosis of the disease but are not valued by the doctor. Many of these patients prefer to seek a doctor of their confidence, whom they can reliably trust their illness and the symptoms they suffer from. To overcome these difficulties, the author suggests that the physician has first to try understanding the patient's way of life and seeing how he interprets the disease before starting a medical investigation. The process of establishing human relationships with patients contributes to developing the physician's sense of responsibility, as well as improving outcomes and adherence to treatment, increasing the degree of patient satisfaction. William Osler stated that the patient should work in the anamnesis as a text, so he suggested medical professors that they extend the contact of their students with the patient and thus avoid a too theoretical medical culture. The consequences of a misunderstanding between these two models the one of the doctor and the patient's one may also lead to litigation, in a large extent for not having had a proper communication than the consequence of a possible medical error.

There are two latent dimensions of the doctor-patient relationship, which offers no room for patients to express themselves with more autonomy, and also does not contribute for therapeutic adherence. ${ }^{7}$

They are: 1) Generic patient trust in the doctor that we may call the Mythical Relationship 2) Difficulty and lack of patient choices, to which physicians are not sensitive - Model between the mythical and the paternalistic. On the other hand, the way in which medical / patient communication takes place has Implications in adherence to therapy, and is at risk of litigation due to lack of information. Without pretending to make the physician - patient relationship methodology a mere check list, because it seems to be too instrumental, we suggest some practical readings that may facilitate the dialogue and communication between the two parties. Thus the medical practice that was based on Pathophysiology, Deductive Reasoning and Clinical Experience underwent a radical change with the image boom. Lobo Antunes recalls that the disease changed its meaning with access to this new methodology, and it was seen as a clone or the "double" of the disease. We then present some practical points facilitating the communication, optimizing the relation concerning the doctor's posture:

a. Compliance and presentation

\section{b. Attention during service}

\section{c. Appropriate language}

As to the Medical Behavior level, the doctor should be considerate, calm, cordial and empathic and avoid treating the patient or family with arrogance or being prejudiced or making jokes about the patient or his illness, devaluing the symptoms.

Discussing in public or changing the voice with the sick person or family members is absolutely prohibited. In the item fulfillment and presentation, the doctor should: Get up and receive the patient at the door, identify himself, call the patient by his name, shake hands and greet the attendants. Reverberating the doctor-patient relationship. The image of the disease can equally be apprehended by both with a reduction of knowledge asymmetry, hegemonically belonging to the physician, the ethical foundation of paternalism. For this reason the behavior and posture of the doctor has also undergone a change.

Paying attention to the patient - the healthcare professional should look the patient in his eyes, pay attention to what is said, not answer the telephone (except in case of emergency), close the office door, avoid interruptions in the consultation, install the patient as well as his companions properly, not move around constantly or leave the room while he is taking care of the patient and not allow other colleagues or staff to systematically enter the room where he is observing the patient.

If the patient does not speak Portuguese nor needs a sign language, an Interpreter or family member should be asked for so as to help in the communication. It is important to let the patient feel free to say what he or she feels like and to naturally guarantee the confidentiality of the information (except in particular third party conditions). As for the Language, this must be appropriate, the doctor should avoid excessive use of medical terms, expressing himself in an appropriate language to the patient and the people who are accompanying him, and adapt his speech to their cultural level. It is important to pause, ask if they understand and if necessary, ask them to explain in their own words. Within the appropriate language and facing a difficult scenario which is to give a bad news taking into account the delicate moment. How to proceed in case of: Serious illnesses, uncured diseases, communicable diseases?

Walter Baile has developed a protocol, SPIKES, which helps the doctor prepare an interview. Thus Walter Baile through this SPIKES protocol corresponding to a mnemonic (Setting up the interview, Perception, Invitation, Knowledge, Emotions, Strategy) teaches its preparation. In a Portuguese translation it would be:

a. Interview preparation - no interruptions.

b. Perception - Trying to perceive patient information about the disease. Correct if wrong

c. If the patient does not want information -? But you want to know treatment plans

d. Information "unfortunately I have bad news" Inform facts slowly. Language without technical terms - COM truth

e. Emotions - Empathic responses. Role of the physician - comforting. Take breaks. Listen closely and Show proximity

f. Plan for the future crucial DISCUSS all treatment options 
Also remember that the doctor / patient / client relationship as today is called in some medical care places has changed, and patients have a posture of recourse to "Dr Google". There are some suggestions for doctors to interact with Internet followers

Thus the doctor MUST: 1 . Try to react positively to the information on the internet, 2. Warn about the variability and quality of the information. 3. Develop strategies to deal with internet information prior to consultation (eg. patients send a summary email before the consultation) 4. Accept that they can find relevant and valid information, previously unknown to themselves

It MUST NOT 1. Be paternalistic or indifferent 2. Be derogatory of comments made by others on the internet. 3. Refuse to accept information found on the Internet. 4. Feeling threatened

Do not forget to register properly in the Clinical Diary, which is an important Information document with legal value, all the accurate and relevant data about the patient, from the date of the consultation, identification, age, sex, city, profession, data record of the consultation (more semiologic details), signature and stamp, reference to which information was provided and obtained informed consent if necessary (in particular in the surgical acts)

Finally, we are pleased to say that in our profession, "in order to achieve the impossible of ourselves ..." as Clarice Lispector would say, there is also a methodology that must be practiced, not in the Messianic sense of divinization of the profession, but as a learned procedure with rules and time and space and working conditions, and peer appreciation for effort and CLEAR.... courage.

Which sometimes divinizes us a little too!

\section{Acknowledgments}

None.

\section{Conflicts of interest}

The author declares no conflict of interest.

\section{References}

1. Cassel EJ. The healer's art: a new approach to the doctor-patient relationship. New York: Lippincott; 1976:47-83.

2. Kleinman A. Patients and Healers in the Context of Culture. Berkeley: University of California Press; 1980:448.

3. Nunes Berta. The Medical Knowledge of the People. Publisher End of Century; 1997.

4. Nunes Berta. Health and disease - the perspective of the patient and the decision to consult. 2000.

5. Nunes JM. Communication in clinical context. Lisbon: Bayer Healthcare; 2007.

6. Kleinman A. Suffering and its professional transformation: Toward an ethnography of interpersonal experience. Culture, Medicine and Psychiatry. 1991;15(3):275.

7. https://www.apifarma.pt/salaimprensa/comunicados/Documents/03\%20 Apresenta $\%$ C3\%A7\%C3\%A3o_Ades $\%$ C3\%A3o\%20\%C3\%A0\%20 Terap $\%$ C3\%AAutica_final.pdf 